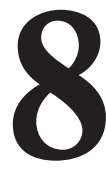

\title{
EL ALUMNADO DEL GRADO DE EDUCACIÓN ANTE LAS TAREAS UNIVERSITARIAS: EMOCIÓN Y COGNICIÓN
}

\author{
(EDUCATION DEGREE STUDENTS DEALING WITH UNIVERSITY TASKS: \\ EMOTION AND COGNITION)
}

Nerea Larruzea-Urkixo

Olga Cardeñoso Ramírez

Nahia Idoiaga Mondragón

Universidad del País Vasco (UPV/EHU)

DOI: 10.5944/educXX1.23453

\section{Cómo referenciar este artículo/How to reference this article:}

Larruzea-Urkixo, N.; Cardeñoso Ramírez, O., e Idoiaga Mondragón, N. (2020). El alumnado del grado de educación ante las tareas universitarias: emoción y cognición. Educación XX1, 23(1), 197-220, doi: 10.5944/educXX1.23453

Larruzea-Urkixo, N.; Cardeñoso Ramírez, O., \& Idoiaga Mondragón, N. (2020). Education degree students dealing with university tasks: emotion and cognition. Educación XX1, 23(1), 197-220, doi: 10.5944/educXX1.23453

\section{RESUMEN}

Las emociones juegan un papel fundamental en los contextos de aprendizaje. Sin embargo, el estudio de las mismas no responde al abanico de situaciones que el alumnado afronta en la universidad, ni profundiza en el componente cognitivo de la emoción en dicho contexto, a pesar de su reconocida importancia. Por ello, este estudio pretende ahondar en la comprensión de las emociones experimentadas por el alumnado ante las situaciones universitarias autopercibidas como significativas, así como en el autodiálogo ligado a dichas emociones. Se analizaron 410 situaciones junto a las emociones y los pensamientos asociados que fueron identificados por 141 estudiantes (102 mujeres y 39 hombres) del Grado en Educación Primaria. La media de edad fue de $20.16, \mathrm{DT}=2.22$, con un rango de edad de 
18 a 35 años. El análisis lexical se llevó a cabo a través del método Reinert que fue complementado a través de un Análisis Factorial de Correspondencia, ambos realizados a través del software Iramuteq. Los resultados revelaron que las emociones más significativas para el alumnado fueron la ansiedad ante presentaciones orales, el enfado principalmente ante tareas grupales y la alegría al obtener buenos resultados tras esforzarse. Esta investigación pone de relieve la necesidad de reconsiderar varios aspectos metodológicos del Grado en Educación Primaria, así como la importancia de incluir la inteligencia emocional en dicha titulación y la utilidad del entrenamiento del alumnado en habilidades de resolución de conflictos, reducción de la ansiedad ante exposiciones orales y habilidades de autorregulación académica.

\section{PALABRAS CLAVE}

Emociones; autodiálogo; educación superior; formación de profesores; inteligencia emocional.

\section{ABSTRACT}

Emotions play a key role in learning experiences. However, little has been studied regarding a) the emotions students experience when dealing with different university situations and b) the cognitive component of emotion in this context, despite its acknowledged importance. For these reasons, this study was aimed at delving into the understanding of students' emotions in self-perceived meaningful situations, as well as the self-talk linked to them. 410 situations - together with the emotions and thoughts related to them - were analyzed, identified by 141 Primary Education Bachelor's Degree students (102 women and 39 men). The average age was $20.16, \mathrm{SD}=2.22$, ranging from 18 to 35. The analysis was carried out through lexical analysis (Reinert method), which was complemented and refined by a Factorial Analysis of Correspondences using Iramuteq software. The results showed that the most significant emotions for students were anxiety when doing oral presentations, anger when working in groups and joy resulting from obtaining good results after making an effort. This research raises the need for reconsidering different methodological aspects of the bachelor's degree, as well as the importance of including emotional intelligence in the same. It also brings to light the relevance of training students in social conflict resolution and anxiety reduction skills together with academic selfregulation abilities. 


\section{KEYWORDS}

Emotions; self-talk; higher education; teacher training; emotional intelligence.

\section{INTRODUCCIÓN}

Las emociones han sido objeto de análisis y preocupación desde la antigüedad (Bisquerra, 2000) ya que poseen un papel fundamental en el bienestar o malestar que las personas manifiestan en todos los ámbitos de sus vidas (Rodrigo-Ruiz, 2016), incluido también en el contexto académico.

La emoción se define como "un estado complejo del organismo caracterizado por una excitación o perturbación que predispone una respuesta organizada (...) que es generada habitualmente como respuesta a un acontecimiento externo o interno" (Bisquerra, 2000: 48). En cuanto a su clasificación, existen diversidad de teorías y propuestas, pero todas ellas se sustentan en un eje que va desde el placer al displacer (Bisquerra, 2009). Es decir, podemos experimentar emociones positivas y negativas (RodrigoRuiz, 2016) ${ }^{1}$. Las positivas son agradables, se sienten al alcanzar una meta y el afrontamiento de dicha meta se centra en el disfrute. Por el contrario, las emociones negativas son desagradables, se generan cuando se bloquea o se hallan obstáculos en la consecución de una meta, ante una amenaza o una pérdida (Lazarus, 1990) y alteran el estado de bienestar. Siguiendo esta distribución y tomando en consideración la perspectiva de las familias de emociones $^{2}$, las principales emociones negativas son a) el miedo, es decir, la emoción experimentada ante un peligro o amenaza; b) la ira, entendida como una reacción de irritación, furia o cólera generada por la indignación de sentirse perjudicado/a; c) la tristeza producida por la pérdida de algo valorado como importante; d) el asco comprendido como el rechazo a algo o a alguien debido a estímulos desagradables, e) la ansiedad, es decir, la emoción generada por una preocupación o anticipación de un peligro futuro e imprevisible y f) la vergüenza, emoción social desencadenada por el sentimiento de pérdida de dignidad o humillación. Por otro lado, las emociones positivas incluyen a) la alegría producida por un suceso favorable, b) el amor entendido como el afecto hacia otra persona, animal o cosa generado por valoraciones subjetivas, y c) la felicidad o la valoración global de la vida, aspecto que mantiene cierta estabilidad temporal (Bisquerra, 2000, 2009).

Esta investigación profundiza en el aspecto cognitivo de la emoción ya que el hecho de que una emoción sea positiva o negativa se sustenta y 
se mantiene en los pensamientos asociados a ella. Es decir, la emoción que experimenta una persona está sujeta a la evaluación o valoración automática (appraisal) que realiza sobre dicha experiencia, acontecimiento o situación concreta (Arnold, 1960). Tal y como explicaba la famosa idea del filósofo griego Epicteto y en la que posteriormente Ellis (1980) basó su teoría, una persona no se siente perturbada por los hechos en sí mismos, sino por la visión que tiene de ellos. Esta interpretación o valoración de una situación concreta está estrechamente ligada al autodiálogo o diálogo interno de las personas, es decir, a la conversación interior que mantienen en su vida cotidiana y que propiciará unas emociones u otras. Así, un pensamiento positivo, conducirá a una emoción positiva y un pensamiento negativo incitará a sentir una emoción negativa. Por ejemplo, cuando una persona se enfrenta a una tarea académica concreta como llevar a cabo un trabajo y piensa "No puedo hacerlo, es demasiado para mí" experimentará emociones como la tristeza o frustración. Por el contrario, si se dice a sí misma “iQué ganas tengo de hacerlo! Es un reto muy interesante" se inclinará a sentir emociones positivas como la alegría. Es más, pensamientos autorregulatorios como "Es difícil, pero si me esfuerzo y me concentro, lo lograré" pueden incluso ayudarle a enfrentar la tarea controlando su emoción y consecuentemente, su desempeño en la misma. Por lo tanto, se puede afirmar que nuestra vida académica y los procesos de aprendizaje están influenciados en gran medida por lo que pensamos y sentimos (Rodrigo-Ruiz, 2016).

Resulta llamativo que a pesar del impacto del diálogo interno en las emociones del alumnado, su estudio en el ámbito universitario sea escaso. De hecho, las investigaciones sobre autodiálogo en este ámbito se han centrado en situaciones concretas como la ansiedad ante los exámenes (Sánchez, Carvajal, y Saggiomo, 2016). No obstante, se debe considerar el trabajo desarrollado por Calvete, et al., (2005) ya que, a pesar de ser parte de un cuestionario de autodiálogo de la vida en su globalidad, mostró algunas situaciones relativas a la universidad y se trata del único estudio que profundiza en el autodiálogo en situaciones propias de la realidad universitaria en nuestro territorio hasta la actualidad (Villa, Calvete, Villardón, y Cardeñoso, 2005). Concretamente, se centró en la obtención de una buena calificación tras esforzarse para un examen, la falta de implicación del estudiantado en los trabajos en equipo y la realización de presentaciones orales. Debido a su aportación al estudio del autodiálogo en diversas situaciones que acontecen en la universidad, la mencionada investigación será un importante referente en el presente trabajo.

Atendiendo a las investigaciones sobre emociones en contextos académicos, en primer lugar, cabe destacar que la gran mayoría de estudios abordan la ansiedad ante exámenes (Pekrun, Goetz, Titz, \& Perry, 2002; Rowe \& Fitness, 2018), tanto a nivel internacional (Furlan, Ferrero, \& 
Galart, 2014; Furlan, Piemontesi, Heredia, \& Sánchez, 2015) como en el territorio español (Mayo, González-Freire, y Moreira, 2015). No obstante, varios trabajos también muestran emociones relacionadas con el estudio en casa o el hecho de estar en clase (Pekrun, Goetz, Titz, \& Perry, 2002), el afrontamiento de situaciones complejas (Corsini, Bustos, Fuentes, \& Cantín, 2012) o a la producción oral en inglés (Celina \& Sánchez, 2016).

Si bien la ansiedad ante exámenes es la emoción explorada en mayor medida, el estudio de otras emociones negativas, como el aburrimiento o la desgana, ha aumentado en los últimos años en el panorama internacional (Bench, 2014; Pekrun, Hall, Goetz, \& Perry 2014; Pekrun, \& Perry, 2014) y también a nivel nacional (Marcos-Merino, 2019).

Sin embargo, los procesos de aprendizaje también nos pueden llevar a experimentar emociones positivas como la alegría o el placer (Christie, Tett, Cree, Hounsell, \& McCune, 2008; López-Cassá, Pérez-Escoda, y Alegre, 2018). Ibáñez (2002) señaló que las emociones positivas o favorables del alumnado estuvieron ligadas a aspectos como sentir motivación por las clases, tratar contenidos contextualizados en las propias experiencias, sentirse valorado/a como estudiante o poder participar o confrontar distintos puntos de vista. Por su parte, Mega, Ronconi, y De Beni (2014) mostraron que las emociones positivas tenían mayor influencia en la motivación y estrategias autorregulatorias del alumnado que las emociones negativas y que estas eran predictoras de diversos componentes de autorregulación. La investigación desarrollada en España también apoya estos resultados (Marcos-Merino, 2019), ya que estudios recientes mostraron que las emociones positivas fueron identificadas por el alumnado incluso en mayor medida (Retana-Alvarado, de las Heras, Vázquez-Bernal, y Jiménez-Pérez, 2018) y eran experimentadas con mayor intensidad (Sáez de Ocáriz, Lavega, Mateu, y Rovira, 2014) que las emociones negativas. En el reciente estudio de Marcos-Merino (2019), con una muestra similar a la de este trabajo, se obtuvo que la alegría, el entusiasmo y la satisfacción estaban relacionadas con la práctica y los resultados de aprendizaje. Además, en esta línea que hace hincapié en el papel del autodiálogo en la regulación del aprendizaje, Suárez-Riviero y Fernández-Suárez (2013) mostraron que la estrategia motivacional-afectiva más utilizada era la de autorrefuerzo, conformada por los halagos o recompensas que el propio alumnado hace sobre su propia capacidad a sí mismo.

En cualquier caso, tal y como manifiesta Paoloni (2014: 571) "la investigación sobre emociones en contextos de aprendizaje constituye una necesidad actual dentro del campo de estudio de la Psicología Educacional" dado que su estudio emerge lentamente y de forma segmentada (Schutz \& Pekrun, 2007), es decir, considerando cada emoción aisladamente. 
Es más, resultaría clave indagar, no solo en las emociones, sino también en los pensamientos ligados a ellas y referidas a las situaciones reales experimentadas por el alumnado en el contexto universitario actual.

La utilidad de conocer la manera de sentir del alumnado cobra aún mayor relevancia cuando se trata del futuro profesorado, ya que la enseñanza es una actividad emocional (Hargreaves, 2001) en la que los procesos de aprendizaje del alumnado están altamente influenciados por las emociones de sus docentes. En concreto, las emociones negativas tendrán un impacto negativo en el alumnado, mientras que las positivas influenciarán positivamente su aprendizaje (Pekrun, et. al., 2002). De hecho, las emociones que experimenta el profesorado son transmitidas a sus estudiantes y pueden repercutir en su conducta (Becker, Goetz, Morger, \& Rallenucci, 2014; Rodrigo-Ruiz, 2016) e incluso en su desempeño académico (Beilock, Gunderson, Ramirez, \& Levine, 2010).

Por ello, dada la necesidad de ahondar en las emociones del alumnado de educación de una manera contextualizada en sus propias vivencias y desde una perspectiva holística que incluya las situacionesy los pensamientos asociados a las mismas, este estudio propone los siguientes objetivos: 1) explorar las situaciones significativas que generan emociones para el alumnado de educación; 2) conocer cuáles son las emociones negativas y el autodiálogo asociado a las mismas; y 3) conocer las emociones positivas y el autodiálogo ligado a ellas. En base a estos objetivos, se proponen tres hipótesis: 1) se espera hallar situaciones de aprendizaje relacionadas con los diferentes niveles de implicación en los trabajos en equipo, la obtención de una buena calificación tras esforzarse y el afrontamiento de exposiciones orales; 2) se espera hallar la emoción y autodiálogo negativos relativos a la ansiedad ante los exámenes; y 3) se espera hallar emociones y autodiálogo positivos como la alegría y la satisfacción en relación a la práctica y resultados académicos. Cabe destacar que no se pueden plantear hipótesis relativas a la relación entre emoción y autodiálogo dado que no existe literatura previa que profundice en el componente cognitivo de la emoción exclusivamente en la universidad.

\section{MÉTODO}

\section{Población y muestra}

La muestra estuvo formada por 141 estudiantes (103 mujeres y 39 hombres) del Grado de Educación Primaria que identificaron un total de 410 situaciones. La media de edad fue de $20.16, \mathrm{DT}=2.22$, con un rango de 
edad que oscilaba entre 18 y 35 años. En lo que respecta a la distribución por cursos, 40 participantes eran de primer curso (30 mujeres y 10 hombres), 53 de segundo curso (42 mujeres y 11 hombres), 26 de tercero (15 mujeres y 11 hombres) y 22 (16 hombres y 6 mujeres) de cuarto curso. La información fue recogida entre los meses de marzo y mayo de 2017 y las pruebas que se describirán a continuación tuvieron una duración aproximada de treinta minutos. Este estudio fue aprobado por el Comité de Ética en la Investigación con Seres Humanos (CEISH) de la Universidad del País Vasco (UPV-EHU).

\section{Instrumentos}

Dada la complejidad a la hora de evaluar situaciones del ámbito emocional (Pulido-Acosta \& Herrera-Clavero, 2019), se decidió optar por las técnicas cualitativas que se describirán a continuación ya que son consideradas efectivas para desarrollar estudios que ahonden en el conocimiento de la emoción de las personas (Rowe \& Fitness, 2018).

\section{Identificación de situaciones}

En primer lugar, cada participante describió tres situaciones de la vida académica universitaria en las que recientemente experimentó emociones intensas y especificó la emoción generada en cada situación. Se pretendió analizar la realidad de sus emociones de manera contextualizada en su experiencia académica, evitando el sesgo de analizar las emociones de manera aislada o fragmentada (Schutz \& Pekrun, 2007). Asimismo, cabe destacar que se decidió que el estudiantado identificase las situaciones por sí mismo, ya que cuando la situación o el estímulo es relevante para la persona provoca mayor respuesta cognitiva y se obtienen más pensamientos (Cacioppo \& Petty, 1981).

\section{Listado de pensamientos}

Con el fin de conocer dichos pensamientos, tras una explicación conceptual sobre el significado del autodiálogo para que supiesen identificarlo adecuadamente, se solicitó al alumnado que anotase los autodiálogos que tenía en cada una de las situaciones anteriormente seleccionadas mediante la técnica de listado de pensamientos. Esta técnica consiste en solicitar a las personas participantes que anoten todos los pensamientos, ideas o reflexiones que han tenido ante situaciones específicas (Igartua, 1998; Morin, Duhnych, \& Racy, 2018). 
Este procedimiento posee la ventaja de ser privado y de no intimidar a los participantes. Además, no restringe las dimensiones obtenidas, es decir, no fuerza al alumnado a clasificar su autodiálogo en categorías o contenidos ya existentes como ocurre en el caso de cuestionarios de autoinforme (Morin, Duhnych, \& Racy, 2018). Asimismo, este método es susceptible a modificaciones en el entorno y es útil a la hora de considerar diferencias individuales (Cacioppo \& Petty, 1981), por lo que posibilita la detección de cambios producidos en el nuevo contexto universitario e incluye la variabilidad individual en función de los datos que se describen en el próximo apartado.

\section{Otros datos de interés}

Además de la información obtenida a través de las pruebas descritas previamente, también se solicitó al estudiantado que indicase su género, el año de grado que estaba cursando y su rendimiento académico. Este último aspecto se evaluó a través de la nota media del grado definida operativamente como media aritmética de las calificaciones obtenidas en todas las asignaturas de grado hasta el momento de la cumplimentación de la prueba.

\section{Procedimiento de recogida y análisis de los datos}

A fin de realizar unos análisis de contenido de las situaciones y de los pensamientos y emociones asociados a ellas, se hizo uso del análisis lexical mediante el método Reinert a través del software Iramuteq. Este análisis radica en la idea de que todo discurso es expresado mediante un sistema de mundos lexicales que organiza una racionalidad y da coherencia a todo lo que los y las participantes enuncian. Un mundo lexical está formado por el grupo depalabras que constituyen un fragmento del discurso. El objetivo del algoritmo del software es el de mostrar, a través del análisis de situaciones, pensamientos y emociones, los mundos lexicales o representaciones sociales usualmente compartidos por las personas participantes (Idoiaga, Gil de Montes, y Valencia, 2016; Ormeño, 2016).

Se analizaron un total de 410 situaciones con sus respectivos pensamientos y emociones. En primer lugar, el software hace una división entre palabras completas (sustantivos, verbos, adjetivos y adverbios) y palabras herramienta (artículos, preposiciones, conjunciones y pronombres) para incluir en el análisis únicamente las palabras completas. Asimismo, el corpus inicial es dividido en Unidades Contextuales Elementales o ECUs, las cuales tienen una longitud aproximada 30 a 50 palabras. De este modo, el 
corpus se analiza considerando qué palabras completas hay en cada ECU. Estas palabras son utilizadas para crear una tabla de contingencia que muestra la distribución del vocabulario por ECU. Partiendo de esta tabla de contingencia, el programa genera una matriz cuadrada de distancias, lo que implica que dos ECUs estarán cerca entre sí, si comparten algunas de las palabras analizadas (Reinert, 1996).

A continuación, de acuerdo con el método Reinert, se realiza un análisis jerárquico descendente de distancias para clasificar y agrupar las ECUS en clases y conocer el vocabulario más característico de cada una de ellas. Mediante este proceso, el software ayuda a la interpretación de textos, ya que extrae clases o grupos de palabras, que ocurren simultáneamente y están diferenciadas del resto. Los criterios para la inclusión de cada forma en sus respectivas clases, serían la frecuencia superior al promedio de ocurrencias en el corpus, así como la asociación con la clase determinada por el valor de chi-cuadrado igual o superior a 3 . Esto proporciona un margen de error $<0,05$ (de un grado de libertad =1) (Ormeño, 2016).

Así, se obtuvieron una serie de clases formadas por palabras y segmentos de texto (ECUs) típicos con valores estadísticos significativos. Esto proporcionó la base estadística para interpretar las clases como mundos lexicales o temáticos generales. Las operaciones en el método Reinert son estadísticas, transparentes y reproducibles, hasta el momento de la interpretación, en el que las investigadoras asignan una etiqueta a cada clase basándose en el vocabulario específico y segmentos típicos de la misma propuestos por el software, basándose en coocurrencias y patrones de distribución (Schonhardt-Bailey, 2013).

El método Reinert también calcula la relación entre cada temática general o clase y las variables independientes utilizando tests de independencia. Si existe una proporción de ECUs significativamente mayor perteneciente a una clase donde el nivel de una variable independiente es mayor que el resto de niveles combinados, se considera que la clase estará asociada a esa variable independiente (Klein \& Licata, 2003). El software realiza el mismo análisis para las otras clases y niveles de las variables independientes. En esta investigación se hizo una diferenciación entre a) el género, b) el curso del grado (primero, segundo, tercero o cuarto) y c) el rendimiento académico (alto (8-10), medio (7-8) o bajo (6-7).

Con el fin de indagar en los mundos lexicales y representaciones sociales obtenidas, además del Análisis Jerárquico Descendente (método Reinert), se llevó a cabo el Análisis Factorial de Correspondencia (AFC). Este análisis se basa en cálculos de la nube de palabras que constituye un corpus y determina los factores sobre los cuales se distribuyen las clases 
de palabras estadísticamente independientes (formas). El concepto de pertenencia a una clase se basa en la distancia a un eje de inercia y sus representaciones gráficas son bidimensionales, dividiéndose en dos factores (Ormeño, 2016). Así, este análisis aporta precisión al Análisis Jerárquico Descendente dado que permite observar la correspondencia de las formas según su posición en los ejes.

\section{RESULTADOS}

Como se ha mencionado previamente, las situaciones, emociones y pensamientos identificados por el alumnado fueron analizadas mediante el método Reinert a través del software Iramuteq. El corpus completo estuvo formado por 15.339 palabras y 2.014 de ellas eran palabras únicas. El análisis jerárquico descendente segmentó el texto en 492 ECUs.

\section{Análisis Jerárquico Descendente (CDH)}

Como se puede observar en el dendograma de la Figura 1, siguiendo la división del análisis de clúster, se extrajeron cinco clases o temáticas principales referidas a diferentes emociones intensas que vive el alumnado universitario.

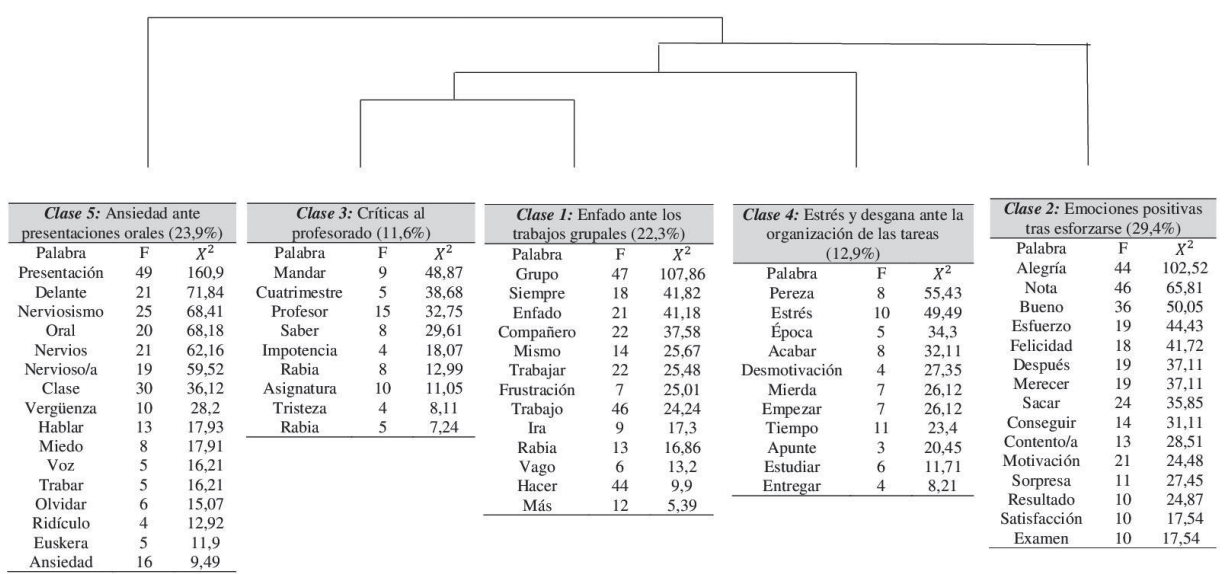

Figura 1. Dendrograma de la Clasificación Jerárquica Descendente

La primera clase "Enfado ante los trabajos grupales" (22.26\%) mostró emociones como la ira $\left(\mathrm{X}^{2}=17.3\right)$ o el enfado $\left(\mathrm{X}^{2}=41.18\right)$, la frustración $\left(X^{2}=25.01\right)$ y la rabia $\left(X^{2}=16.86\right)$ a la hora de trabajar en grupo. Así, el 
alumnado expresó pensamientos concretos ligados a estas emociones debido a conflictos relacionados con los distintos niveles de implicación en los trabajos grupales: "Mis compañeros de grupo no tienen ganas de trabajar ni ningún interés por el grado. No tienen en cuenta la carga de trabajo para casa que supone el no trabajar" (mujer, segundo curso, rendimiento académico normal; $\mathrm{X}^{2}=260.56$ ); "Son unos vagos de mierda, nunca hacen nada. Siempre me toca a mí hacer más que el resto y luego me ponen excusas. Ya verán en el siguiente trabajo, se van a enterar" (hombre, primer curso, rendimiento académico alto; $\mathrm{X}^{2}=292.35$ ); "El trabajo se tiene que repartir pero no todo el mundo hace su parte y siempre hay alguien que pone excusas. Yo he sacado tiempo de debajo de las piedras y aquí la gente se pasa por el forro tener que hacer el trabajo. Nos afecta a todos que esa persona no haya hecho el trabajo y además tendremos que hacer trabajo extra por su culpa" (mujer, primer curso, rendimiento académico normal; $\left.X^{2}=242.22\right)$. Por el contrario, a pesar de que hayan sido menos frecuentes, el alumnado menos implicado también expresa su desagrado ante los trabajos en grupo: "No me es agradable trabajar en grupo y cuanto mayor sea el grupo menos. Tengo mi manera y ritmo de trabajar y el objetivo que me pongo es llegar al 5 . Tener que adaptarme al ritmo de mis compañeros que quieren más nota me resulta incómodo y estresante" (mujer, segundo curso, rendimiento académico normal; $\mathrm{X}^{2}=211.43$ ). Además, algunos estudiantes reflejaban pensamientos centrados en decisiones que tomarían en el futuro para cambiar la situación: "El grupo de trabajo no funciona bien pero son mis amigos. Suele haber conflictos entre gente del grupo y al final da la sensación de que terminamos haciendo siempre el trabajo los mismos. Quiero dejar el grupo. El año que viene tengo que cambiar" (mujer, tercer curso, rendimiento académico normal; $\mathrm{X}^{2}=303.13$ ). Esta clase estaba significativamente relacionada con el alumnado de primer curso $(\mathrm{p}=.008)$ y con el alumnado de rendimiento académico normal ( $\mathrm{p}=.001)$.

La clase 2, denominada "Emociones positivas tras esforzarse" $(29.35 \%)$, reflejó emociones positivas como la alegría $\left(X^{2}=102.52\right)$, la felicidad $\left(X^{2}=41.72\right)$, la motivación $\left(X^{2}=24.48\right)$, la satisfacción $\left(X^{2}=17.54\right)$ y el orgullo $\left(X^{2}=10.97\right)$ del alumnado tras haber llevado a cabo de manera exitosa una tarea que requería esfuerzo. Estas situaciones estaban ligadas a distintas tareas universitarias como la obtención de buenas calificaciones en exámenes, proyectos o trabajos y el alumnado expresó las mencionadas emociones a través de pensamientos positivos: “iSoy genial! Me lo merezco, con todo lo que he trabajado... Voy a llamar a mi madre" (mujer, cuarto curso, rendimiento académico alto; $\mathrm{X}^{2}=326$ ); “ßBuen trabajo, Pablo! Tienes que seguir trabajando para conseguir tus objetivos" (hombre, cuarto curso, rendimiento académico normal; $\left.\mathrm{X}^{2}=285.54\right) ; “ ¡ N o$ es posible! ¿Matrícula de honor yo? ¡No me lo puedo creer! Mi esfuerzo ha servido para algo" (mujer, tercer curso, rendimiento académico alto; $\mathrm{X}^{2}=174.14$ ). También 
se hallaron pensamientos relacionados con valoraciones del proceso: " $\mathrm{He}$ sacado buena nota. La próxima vez no voy a estresarme tanto que no sirve de nada y solo me hace sentirme mal" (mujer, primer curso, rendimiento académico normal; $\mathrm{X}^{2}=288.07$ ); "Al final con esfuerzo todo se consigue. Ha valido la pena todo lo que he estudiado" (mujer, segundo curso, rendimiento académico normal; $\left.\mathrm{X}^{2}=283.55\right)$. Esta clase estuvo significativamente relacionada con el alumnado de segundo curso $(\mathrm{p}=.024)$.

En lo que respecta a la clase 3 "Críticas al profesorado" (11.61\%), el alumnado expresó su impotencia $\left(X^{2}=18.07\right)$, rabia $\left(X^{2}=12.99\right)$, tristeza $\left(\mathrm{X}^{2}=8.11\right)$ e incluso asco $\left(\mathrm{X}^{2}=8.95\right)$ ante diferentes aspectos atribuidos al profesorado universitario. Estas quejas se centraban principalmente en la excesiva carga de trabajos encomendados a corto plazo: "Mandan un trabajo nuevo cuando queda una semana por terminar el curso, ¿no podían haberlo explicado antes? No me creo que no se den cuenta de que nos saturan a trabajos. Y, por si fuera poco, lo hacen cuando se nos acercan los exámenes..." (mujer, segundo, rendimiento académico normal; $\mathrm{X}^{2}=$ 186,39); "Nos mandan hacer trabajos en grupo constantemente en todas las asignaturas y a veces sin poder elegir tu grupo, ojalá se dieran cuenta los profesores de que no por mandar más se aprende más. Siento estrés cuando todos se acumulan" (mujer, primero, rendimiento académico alto; $X^{2}=186,39$ ). También expresaban su desagrado sobre otras cuestiones metodológicas en las que sienten que pierden el tiempo a través de los siguientes pensamientos: "Lunes, primera clase y el profesor lleva dos horas y media hablando (...) me pregunto a mí mismo a ver qué hago en clase, me parece que estoy perdiendo el tiempo y perder el tiempo que no vamos a recuperar me genera desagrado" (hombre, primer curso, rendimiento académico sin especificar; $\mathrm{X}^{2}=125.75$ ); "Para hacer lo que hace esta ya me pongo yo ahí. Es vergonzoso, en la próxima clase le voy a preguntar a ver qué va a hacer porque para no hacer nada no vengo. Yo también tengo muchas cosas y no estoy para perder el tiempo" (mujer, segundo curso, rendimiento académico alto; $\left.\mathrm{X}^{2}=103.76\right)$. Esta clase se halló relacionada significativamente al alumnado que no especificó su rendimiento académico $(\mathrm{p}=.003)$. No se hallaron diferencias por género o curso.

La clase 4 "Estrés y desgana ante la organización de las tareas" $(12,57 \%)$ mostró situaciones relacionadas con la desgana o pereza $\left(\mathrm{X}^{2}=\right.$ 55.49), la desmotivación $\left(X^{2}=27.35\right)$ o el estrés $\left(X^{2}=49.49\right)$ a la hora de organizarse para llevar a cabo tareas como empezar a estudiar o realizar un trabajo. Por un lado, el alumnado expresó su desidia mediante los siguientes pensamientos negativos: "Mierda, tengo que recopilar todos los apuntes, bajarme los archivos que contienen la teoría uno a uno y clasificarlos. Pero sobre todo tengo que ponerme a estudiar y a mí lo que me gusta es trabajar" (hombre, tercer curso, rendimiento académico alto; $\mathrm{X}^{2}=135,71$ ); "Tengo un 
trabajo gordo y ando justo de tiempo. No tengo ganas de hacerlo y pienso que es inútil. ¿Para qué voy a hacer esa mierda? No lo quiero hacer... ¡Cuánto mejor estaría ahora en la calle! Preferiría estar entrenando ahora” (hombre, tercer curso, rendimiento académico normal; $X^{2}=132,10$ ). Por otro lado, también se hallaron pensamientos negativos que reflejaban el estrés del alumnado: “¡Cuántas cosas tengo que hacer! ¡Ay, que no me va a dar tiempo!" (mujer, segundo curso, rendimiento académico alto, $\mathrm{X}^{2}=$ 126.34); "No tengo tiempo para hacer todo. No sé cómo me voy a organizar" (mujer, primer curso, rendimiento académico normal, $\mathrm{X}^{2}=115.39$ ); “Tendría que haber empezado antes. Tengo poco tiempo para hacer demasiadas cosas. No me va a dar tiempo" (mujer, tercer curso, rendimiento académico normal; $X^{2}=97.28$ ). La clase 4 estuvo significativamente relacionada con el alumnado de tercer curso y rendimiento académico normal.

Finalmente, la clase $5(23.87 \%)$ denominada "Ansiedad ante presentaciones orales” reflejó la ansiedad o nerviosismo $\left(\mathrm{X}^{2}=86.21\right)$, vergüenza $\left(\mathrm{X}^{2}=28.2\right)$ y miedo $\left(\mathrm{X}^{2}=17.91\right)$ del alumnado a la hora de llevar a cabo exposiciones delante sus compañeros. Así, se hallaron pensamientos negativos relacionados con la ansiedad centrados en juicios o valoraciones de los demás: "Lo voy a hacer mal y seguramente me haga un lío con lo que tenga que decir y la gente piense que lo hago fatal" (mujer, primer curso, rendimiento académico normal; $\mathrm{X}^{2}=442.28$ ); "Me va a temblar la voz. Se me va a olvidar lo que tengo que decir. A ver qué piensa la gente de mí..." (mujer, segundo curso, rendimiento académico alto; $X^{2}=395.31$ ); "No lo quiero hacer, me voy a trabar. Se me va a olvidar. No quiero estar nerviosa. Me lo van a notar. ¡Socorro!”(mujer, segundo curso, rendimiento académico alto; $\left.\mathrm{X}^{2}=391.25\right)$; "Lo voy a hacer fatal. Todo el mundo se va a reír de mi presentación. Me voy a quedar en blanco. No voy a saber expresarme correctamente, me voy a poner roja y se van a dar cuenta" (mujer, primer curso, rendimiento académico normal; $X^{2}=341.92$ ). Además, varios participantes identificaron la ansiedad ante presentaciones orales cuando se trataba de otra lengua distinta a la de uso habitual: "Vaya chopo parece que no tengo ni idea de euskera, me cago en los verbos" (hombre, segundo curso, rendimiento académico normal; $\mathrm{X}^{2}=241.13$ ); "Sobre todo en inglés (...) ¡Qué vergüenza! Todo el mundo mirándome... Seguro que la cago y hago el ridículo" (mujer, primer curso, rendimiento académico alto; $\left.\mathrm{X}^{2}=353.21\right)$; "Joer, no sé hablar bien en castellano. Menudas cagadas voy a hacer" (mujer, cuarto curso, rendimiento académico normal; $X^{2}=286.92$ ). No obstante, cabe destacar que parte del alumnado hacía uso de pensamientos para afrontar y controlar la situación y reducir esa ansiedad: “iQué nervios! Pero te va a salir bien, Paula. ¿Y si me quedo en blanco? Bueno, ya está, no pienses más" (mujer, segundo curso, rendimiento académico normal; $\mathrm{X}^{2}=$ 366.90). 


\section{Análisis Factorial de Correspondencia (AFC)}

El Análisis Factorial de Correspondencia refuerza y complementa estos resultados dado que categoriza la valencia de las emociones en un espectro que va de las positivas a las negativas, así como categoriza el tipo de tareas según si son individuales o colectivas. La figura 2 muestra esta combinación de una manera gráfica.

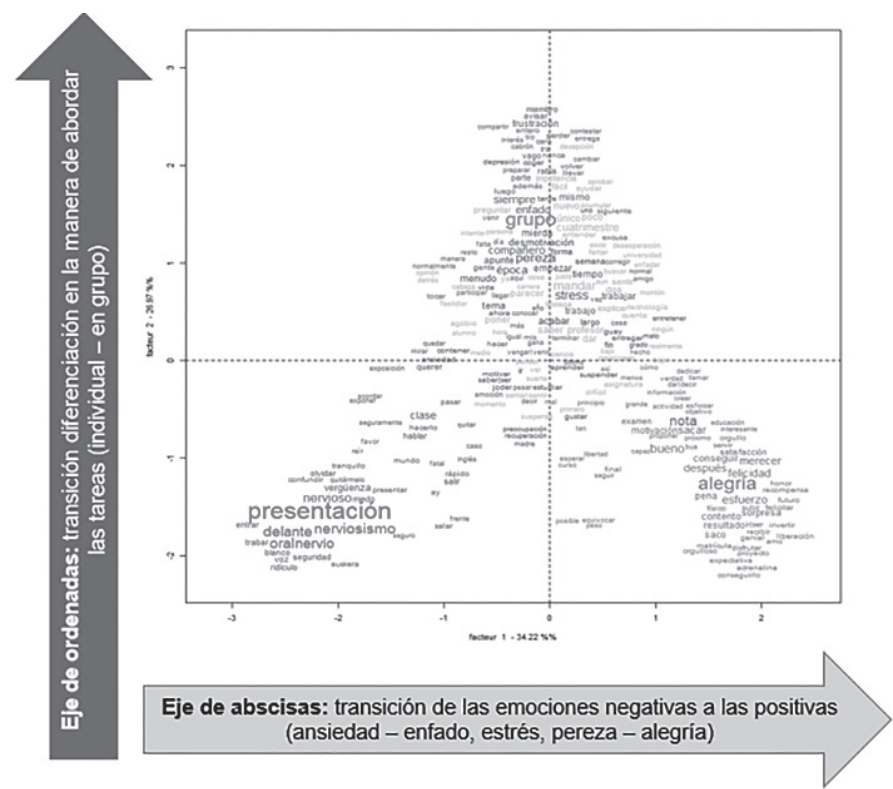

Figura 2. Análisis Factorial de Correspondencia de las emociones, situaciones y pensamientos en el contexto universitario

Si se pone atención en el eje de abscisas, se observará que el primer factor, compuesto por el $34,22 \%$ de la masa total del corpus, dividió las clases obtenidas en función de las emociones. Así, separó la clase 5 "Ansiedad ante presentaciones orales" (situada en las abscisas negativas) de la clase 2 "Alegría tras esforzarse" (abscisas positivas) y ambas se distinguen de las clases 1, 3 y 4 tendentes a la centralización, situándose en torno al eje de abscisas. Se puede observar la transición de emociones negativas al realizar presentaciones orales relacionadas con la ansiedad ("nerviosismo", "nervios", "nervioso/a") hasta las positivas, en la que predomina la "alegría" y se relaciona con la "nota" y el "esfuerzo". En este transcurso de la ansiedad a la alegría, se hallan emociones negativas como el "enfado" el "estrés" o la "pereza".

El segundo factor $(26,97 \%)$ se centró en las tareas académicas y la manera de abordarlas, individualmente o en grupo, tal y como se extrae al 
examinar el eje de ordenadas. Las clases 1, 3 y 4, situadas en su mayoría en las ordenadas positivas, aparecen claramente separadas de las clases 2 y 5 y tratan sobre de tareas realizadas en "grupo". Las clases 1 y 5 (ordenadas negativas) se ocupan de tareas realizadas individualmente, concretamente, la realización de "presentaciones" orales y la obtención de buenas notas tras el esfuerzo personal, respectivamente.

Por lo tanto, analizando los resultados considerando la combinación de estos factores, se puede deducir que las emociones negativas principales serían por un lado, la ansiedad al llevar a cabo presentaciones orales, tarea a realizar de manera individual y por el otro, la ira, estrés o desgana al llevar a cabo tareas en grupo. En lo que respecta a las emociones positivas, se relacionan con el esfuerzo personal e individual y la obtención de buenas calificaciones.

\section{DISCUSIÓN}

Esta investigación, tras el análisis de las 410 situaciones generadas por 141 estudiantes, supone un primer acercamiento al abanico global de emociones experimentadas por el futuro profesorado en su vida universitaria, así como a los pensamientos y situaciones concretas asociados con cada una de ellas. Atendiendo a los objetivos e hipótesis propuestos y tomando en consideración el análisis jerárquico descendente, este apartado se estructurará en función de dichos objetivos.

El primer objetivo de este trabajo era explorar las situaciones significativas que generasen emociones para el alumnado de educación, partiendo de la hipótesis que estimaba que se hallarían situaciones relacionadas con los diferentes niveles de implicación en los trabajos en equipo, la obtención de una elevada y satisfactoria calificación tras esforzarse y el afrontamiento de exposiciones orales siguiendo lo obtenido en el estudio de Calvete, et al., (2005). Así, los resultados de este trabajo son muy similares a los mostrados en la investigación de referencia y se puede afirmar que la hipótesis planteada fue confirmada tal y como mostraron las clases 1 (enfado ante los trabajos grupales), 2 (emociones positivas tras esforzarse) y 5 (ansiedad ante presentaciones orales). Cabe destacar que la información que se esperaba hallar fue completada por nuevas situaciones, concretamente por las clases 3 (críticas al profesorado) y 4 (estrés y desgana ante la organización de las tareas). El hecho de haber obtenido información adicional puede ser debido al momento histórico y a que el trabajo de Calvete, et al. (2005) incluía situaciones de la vida cotidiana, no siendo específico exclusivamente del ámbito académico. 
Un segundo objetivo del estudio era conocer cuáles eran las emociones negativas y el autodiálogo asociado a las mismas y se postulaba la hipótesis de que se hallaría la ansiedad ante exámenes dado que la gran mayoría de estudios al respecto apuntaban en esa dirección tanto a nivel internacional (Furlan, et al., 2014; Furlan, et al., 2015; Pekrun, et al., 2002; Rowe \& Fitness, 2018) como en España (Mayo, González-Freire, y Moreira, 2015). En este trabajo se halló que la emoción negativa identificada con mayor intensidad fue la ansiedad ante presentaciones orales y, aunque en menor medida, el estrés resultante a la acumulación de trabajos y organización de las tareas. Si bien este hallazgo apoya lo obtenido en varios estudios (Calvete, et al., 2005; Celina \& Sánchez, 2016), se opone a la mayoría de investigaciones que ponen el foco en la ansiedad generada por situaciones de examen. En lo referido a los pensamientos relacionados con la ansiedad ante presentaciones, predominaban pensamientos negativos como calificaciones despectivas hacia sí mismos, percepciones de malestar o amenazas internas (confundirse, tartajear al hablar o expresarse de manera concreta en una lengua) y externas, como la evaluación o juicio de sus compañeros. Asimismo, es importante destacar que varios estudiantes trataban de guiar su pensamiento y su conducta de forma positiva para influir en el control y realización de la exposición. El hecho de que fuese significativo en el caso del alumnado de primer curso, puede deberse a la menor frecuencia de tareas que impliquen hablar en público en etapas educativas previas.

Al igual que en el caso del primer objetivo, en este segundo objetivo también se obtuvo información adicional dado que también se hallaron otras emociones negativas. La ira fue la siguiente emoción negativa con más peso para el alumnado. La identificación de esta emoción supone un hallazgo novedoso en el estudio de las emociones en la universidad y estuvo ligada a la situación de trabajo en grupo, a la falta de implicación del estudiantado y a varios aspectos relacionados con el profesorado como la excesiva carga de trabajos en grupo o clases magistrales. A pesar de ser una emoción frecuente en la vida cotidiana, el autodiálogo en relación a esta emoción no ha sido estudiado en profundidad. En este estudio se hallaron pensamientos de venganza o de posibles soluciones futuras para cambiar la situación. Además de la ansiedad y de la ira, el estudiantado también expresó su desgana a la hora de empezar una tarea y organizarse. El estudio de esta emoción también ha sido abordado en varios estudios de los últimos años (Bench, 2014; Marcos-Merino, 2019; Pekrun, et al., 2014; Pekrun \& Perry, 2014).

El tercer y último objetivo de la investigación era determinar cuáles eran las emociones positivas y el autodiálogo ligado a ellas y se proponía la hipótesis de que se esperaban obtener emociones y autodiálogos positivos como la alegría y la satisfacción en relación a la práctica y a los resultados 
académicos. En este trabajo se obtuvo que la clase más frecuente estuvo centrada en emociones positivas, especialmente en la alegría, coincidiendo con lo mostrado en estudios previos (Ibáñez, 2002; Retana-Alvarado, et. al, 2018). Dichas emociones eran resultantes de la satisfacción tras el esfuerzo en la realización de tareas académicas, así como de la obtención de buenas calificaciones en un trabajo u examen. Esta situación de alegría y satisfacción tras conocer una nota favorable en un examen también fue identificada en investigaciones previas (Marcos-Merino, 2019; Villa, et al., 2005). El hecho de que estas emociones también estén relacionadas con trabajos puede ser debido al nuevo modelo universitario en el que ha aumentado el número de tareas grupales Los pensamientos relacionados con esta emoción eran positivos y reflejaban optimismo y orgullo, así como evaluaciones positivas del proceso.

\section{CONCLUSIONES}

Los resultados de esta investigación han proporcionado información específica y novedosa acerca de lo que siente y piensa el alumnado de educación ante las situaciones académicas que considera influyentes en su bienestar o malestar emocional, indagando de esta manera en su mundo cognitivo-emocional.

Se ha mostrado que las emociones positivas pertenecientes a la familia de emociones de la alegría son experimentadas con mayor intensidad que las negativas, a pesar de que desde el punto de vista psicológico (Bisquerra, 2006) y educativo (Paoloni, 2014) hayan sido poco estudiadas. En lo que respecta a las emociones negativas, en primer lugar cabe destacar que si bien la situación que generaba emociones positivas ha sido la más relevante para el alumnado y sobre la que mayor acuerdo existe, el resto de situaciones estaban relacionadas con emociones negativas. Estas se agrupan principalmente en dos de las familias de emociones principales: la ansiedad y la ira.

Por lo tanto, en lo que respecta las implicaciones prácticas del estudio, este trabajo ha puesto de relieve la importancia de dotar al alumnado universitario de estrategias para la reducción de la ansiedad ante exposiciones orales o habilidades de resolución de conflictos sociales, ya que si el estudiantado no resuelve los conflictos en los trabajos en grupo, no logrará los objetivos de aprendizaje cooperativo y constructivo pretendidos, sino que por el contrario se generarán emociones desajustadas relacionadas principalmente con la rabia y la frustración habituales en las dinámicas de grupo (Kitzinger, 2005). En esta línea, también se resalta la relevancia de desarrollar intervenciones en habilidades de regulación en el aprendizaje 
con el fin de superar aspectos motivacionales como la desgana y otros más estratégicos como la organización del trabajo.

Una posible limitación del estudio sería que su alcance se centra en un centro de formación del profesorado. Probablemente, la participación de estudiantes de otras facultades de educación hubiera aportado una visión más amplia. Otra posible limitación sería la medida utilizada para llevar a cabo el estudio. Si bien los métodos cualitativos son indicados para la exploración y acercamiento a las emociones, estos podrían ser completados con medidas cuantitativas. También es preciso mencionar que la muestra, a pesar de que incluye alumnado de los cuatro cursos del grado, es no probabilística por conveniencia. Es importante resaltar la dificultad y el esfuerzo que requiere reclutar alumnado para este tipo de pruebas cualitativas debido al alto nivel de implicación que requieren. Finalmente, el hecho de que el estudiantado fuera del Grado de Educación nos ha permitido conocer la emoción y la cognición del futuro profesorado y su impacto en las aulas. Sin embargo, la recogida de información en alumnado perteneciente a otros grados y ramas de conocimiento hubiera sido útil en aras de establecer comparaciones.

Aun así, este trabajo ha supuesto una novedosa aportación al estudio de las emociones en la universidad. Una de las aportaciones del presente estudio, frente a los citados previamente, sería la consideración del componente cognitivo de la emoción, dado que los mencionados se centran exclusivamente en la dimensión emocional. Asimismo, la investigación desarrollada ofrece una visión holística ya que considera la vivencia de las emociones positivas y negativas y supera el sesgo común del análisis parcelado de cada emoción.

Por lo tanto, la información obtenida en este estudio nos sugiere la importancia de seguir investigando en esta dirección. Partiendo de las limitaciones, el diseño de un cuestionario actual y adaptado a nuestra realidad, además de completar los datos obtenidos en este estudio, posibilitaría las comparaciones entre alumnado de otros grados o territorios ya que el estudio cualitativo de las emociones resulta de gran utilidad a la hora de construir medidas cuantitativas (Rowe \& Fitness, 2018). Asimismo, a partir de lo hallado en esta investigación, sería interesante diseñar y desarrollar programas de reducción de la ansiedad, solución de problemas y aprendizaje autorregulado con el fin de dotar al estudiantado universitario de competencias claves para la ciudadanía en educación actual.

Finalmente, y teniendo en consideración que las emociones que forman parte de la vida académica universitaria, es relevante mencionar la importancia de incluir la inteligencia emocional en los planes de estudio. La literatura existente ha manifestado que altos niveles de inteligencia 
emocional del profesorado, repercuten de manera positiva en diferentes áreas de su trabajo educativo, favoreciendo así no solo al futuro profesorado, sino también al alumnado de nuestras aulas (Rodrigo-Ruiz, 2016). Tal y como manifestó Goleman (1995), están en juego las generaciones futuras.

\section{NOTAS}

1 Existen numerosas corrientes que proponen diferentes clasificaciones de las emociones. Se ha optado por esta propuesta dado que la valencia es un aspecto de las emociones compartido por diversas teorías.

2 Las familias de emociones son clases o conjuntos formados por emociones de la misma especificidad. Las emociones pertenecientes a cada familia se diferencian en matices como la intensidad, la complejidad o simples cuestiones terminológicas (Bisquerra, 2011). 


\section{REFERENCIAS BIBLIOGRÁFICAS}

Arnold, M.B. (1960). Emotion and personality. New York, USA: Columbia University Press.

Beck, J. (1995). Cognitive Therapy: Basics and Beyond. New York, USA: The Guilford Press.

Becker, E.S., Goetz, T., Morger, V., \& Rallenucci, J. (2014). The importance of teachers' emotions and instructional behavior for their students' emotions. An experience sampling analysis. Teaching and Teacher Education, 43, 15-26.

Beilock, S.L., Gunderson, E.A., Ramirez, G., \& Levine, S.C. (2010). Female teachers' math anxiety affects girls' math achievement. Pnas, 107(5), 18601863. doi:_10.1073/pnas.0910967107

Bisquerra, R. (2000). Educación emocional y bienestar. Barcelona: Praxis.

Bisquerra, R. (2009). Psicopedagogía de las emociones. Madrid: Síntesis.

Cacioppo, J.T., \& Petty, R E. (1981). Social psychological procedures for cognitive response assessment: The thought-listing technique. In T.V. Merluzzi, G.R. Glass \& M. Genest (Eds.), Cognitive assessment (pp. 309342). New York, USA: The Guilford Press.

Calvete, E., Estévez, A., Landín, C., Martínez, Y., Cardeñoso, O., Villardón, L., \& Villa, A. (2005). Self-talk and affective problems in college students: Valence of thinking and cognitive content specificity. Spanish Journal of Psychology, 8(1), 56-67.

Celina, M. y Sánchez, A. (2016). Las emociones en la formación inicial de profesores de inglés: Resultados de una prueba piloto con narrativas visuales en la clase de fonética. Educación, Formación e Investigación, 2(4), 2-14.

Christie, H., Tett, L., Cree, V.E., Hounsell, J., \& McCune, V. (2008). A real rollercoaster of confidence and emotions': Learning to be a university student. Studies in Higher Education, 33(5), 567-581. 10.1080/03075070802373040

Corsini, M.G., Bustos, L.M., Fuentes, N J., y Cantín, M. (2012). Niveles de ansiedad en la Comunidad Estudiantil Odontológica. Universidad de La Frontera, Temuco-Chile. Odontostomat, 6(1), 51-57.

Ellis, A. (1980). Razón y emoción en psicoterapia. Bilbao: Desclée de Brouwer.

Furlan, L., Ferrero, M.J., y Gallart, G. (2014). Ansiedad ante los exámenes, procrastinación y síntomas mentales en estudiantes universitarios. Revista Argentina de Ciencias del Comportamiento, 6(3), 31-39.

Furlan, L., Piemontesi, S., Heredia, D., y Sánchez, J. (2015). Ansiedad ante los exámenes en estudiantes universitarios: Correlatos y Tratamiento. Anuario de Investigación de la Facultad de Psicología, 2(1), 3753.

Goleman, D. (1995). Emotional intelligence: Why it can matter more than IQ. New York, USA: Bantam Books.

Hargreaves, A. (2001). Emotional geographies of teaching. Teachers College Record, 103, 1056-1080.

Ibáñez, N. (2002). Las emociones en el aula. Estudios Pedagógicos, 28, 31-45. 
Idoiaga, N., Gil De Montes, L., \& Valencia, J. F. (2016). Communication and representation of risk in health crises: The influence of framing and group identity. Revista De Psicología Social: International Journal of Social Psychology, 31(1), 59-74. 10.1080/02134748.2015.1101313

Igartua, J.J. (1998). La técnica de listado de pensamientos como método de investigación en comunicación publicitaria. Comunicación \& Cultura, 3, 43-62.

Kitzinger, J. (2005). Focus group research: using group dynamics to explore perceptions, experiences and understandings. In I. Holloway (Ed.), Qualitative Research in Health Care (pp. 56-70). Oxford, UK: Blackwell Publishing.

Klein, O., \& Licata, L. (2003). When group representations serve social change: The speeches of patrice lumumba during the congolese decolonization. British Journal of Social Psychology, 42, 571-593. $10.1348 / 014466603322595284$

Lazarus, R. S. (1991). Emotion and adaptation. New York, USA: Oxford University Press.

López-Cassá, E.; Pérez-Escoda, N., y Alegre, A. (2018). Competencia emocional, satisfacción en contextos específicos y satisfacción con la vida en la adolescencia. Revista de Investigación Educativa, 36(1), 57-73. 10.6018/rie.36.1.273131

Mayo, M.A., González-Freire, B., \& Moreira, V. (2015). Ansiedad ante los exámenes en la universidad: Estudio de caso único. Ansiedad y estrés, 21(1), 21-33.

Marcos-Merino, J.M. (2019). Análisis de las relaciones emociones-aprendizaje de maestros en formación inicial con una práctica activa de Biología. Revista Eureka sobre Enseñanza y Divulgación de las Ciencias, 16(1), 1603.

Mega, C., Ronconi, L., \& De Beni, R. (2014). What makes a good student? How emotions, self-regulated learning, and motivation contribute to academic achievement. Journal of Educational Psychology, 106(1), 121-131.

Morin, A., Duhnych, C., \& Racy, F. (2018). Self-reported inner speech use in university students. Applied Cognitive Psychology, 32(3), 376-382. 10.1002/acp.3404

Ormeño, A. (2016). Uso de las tecnologías en el aprendizaje formal, no formal e informal en estudiantes de la carrera de odontología de la universidad de los Andes, Santiago. Chile. [Tesis Doctoral]. Universitat de Barcelona: Barcelona.

Paoloni, R. (2014). Emociones en contextos académicos. Perspectivas teóricas e implicaciones para la práctica educativa en la universidad. Electronic Journal of Research in Educational Psychology, 12(3), 567596. 10.14204/ejrep.34.14082

Pekrun, R., Goetz, T., Titz, W., \& Perry, R. (2002). Academic emotions in students' self-regulated learning and achievement: A program of qualitative and quantitative re-search. Educational Psychologist, 37(2), 91106._10.1207/S15326985EP3702_4

Pekrun, R., Hall, N.C., Goetz, T., \& Perry, R.P. (2014). Boredom and academic achievement: Testing a model of reciprocal causation. Journal of Educational Psychology, 106, 696710.

Pekrun, R. \& Perry, R.P. (2014). Controlvalue theory of achievement emotions. In P.A. Alexander, R. Pekrun, \& L. Linnenbrink-Garcia, (Eds), 
International Handbook of Emotions in Education (pp. 120-141). London, UK: Routledge.

Pulido-Acosta, F., \& Herrera-Clavero, F. (2019). Prediciendo el rendimiento académico infantil a través de la inteligencia emocional. Psicología Educativa, 25, 23-30. _10.5093/ psed2018a16

Reinert, M. (1996). Alceste (version 3.0). Toulouse, France: Images.

Retana-Alvaradoa, D.A., De Las Heras, M.A., Vázquez-Bernal, B., y Jiménez-Pérez, R. (2018). El cambio en las emociones de maestros en formación inicial hacia el clima de aula en una intervención basada en investigación escolar. Revista Eureka Sobre Enseñanza y Divulgación De Las Ciencias, 15(2), 2602-1-2602-16. 10.25267/Rev_Eureka_ensen_divulg_ cienc.2018.v15.i2.2602

Rodrigo-Ruiz, D. (2016). Effect of teachers' emotions on their students: Some evidence. Journal of Education \& Social Policy, 3(4), 73-79.

Rowe, A. D., \& Fitness, J. (2018). Understanding the role of negative emotions in adult learning and achievement: A social functional perspective. Behavioral Sciences, 8(2), 27. $10.3390 / b s 8020027$

Sáez de Ocáriz, U., Lavega, P., Mateu, M., y Rovira, G. (2014). Emociones positivas y educación de la convivencia escolar. Contribución de la expresión motriz cooperativa. Revista de Investigación Educativa, 32(2), 309326. $10.6018 /$ rie.32.2.183911

Sánchez, F., Carvajal, F., y Saggiomo, C. (2016). Autodiálogos y rendimiento académico en estudiantes universitarios. Anales De Psicología, 32(1), 139-147. 10.6018/ analesps.32.1.188441

Schonhardt-Bailey, C. (2013). Deliberating american monetary policy: A textual analysis. Cambridge, MA, USA: MIT Press.

Schutz, P., \& Pekrun, R. (2007). Emotion in education. San Diego, USA: Academic Press.

Suárez-Riveiro, J.M. y FernándezSuárez, A.P. (2013). Un modelo sobre cómo las estrategias motivacionales relacionadas con el componente de afectividad inciden sobre las estrategias cognitivas y metacognitivas. Educación XX1, 16 (2), 231-246. 10.5944/educxx1.16.2.2641

Villa, A., Calvete, E., Villardón, L., y Cardeñoso, O. (2005). Estilos cognitivos y autoconcepto en estudiantes universitarios. Desarrollo de instrumentos de medida e impacto en el ajuste psicológico. Bilbao: Universidad de Deusto - Mensajero. 


\section{PERFIL ACADÉMICO Y PROFESIONAL DE LOS AUTORES}

Nerea Larruzea-Urkixo es doctoranda en el programa Psicodidáctica: Psicología de la Educación y Didácticas Específicas de la Universidad del País Vasco (UPV/EHU), tarea que combina con su labor como Profesora de Educación Primaria. Sus líneas de investigación se centran en el autodiálogo, la autorregulación en el aprendizaje y el impacto del género en las mismas.

Olga Cardeñoso Ramírez. Doctora en Filosofía y Ciencias de la Educación. Profesora agregada en el Departamento de Psicología Evolutiva y de la Educación en la Facultad de Educación de Bilbao de la Universidad del País Vasco (UPV/EHU). Perteneciente a la línea de investigación denominada "Variables Psicoeducativas y Aprendizajes" del Máster y el Doctorado de Psicodidáctica centra una de sus líneas de investigación en el área de "Aprendizaje Autorregulado. Cognición, emoción y género".

Nahia Idoiaga Mondragon. Licenciada en Psicología y Psicopedagogía es doctora en Psicología. Forma parte del grupo de investigación KideON. Enmarcándose en psicología de la educación y la psicología social ha investigado sobre temas ligados a la inclusión socioeducativa, el feminismo y la salud. Actualmente es profesora adjunta en el Departamento de Psicología Evolutiva y de la Educación en la Facultad de Educación de Bilbao de la Universidad del País Vasco (UPV/EHU).

Dirección de los autores: Nerea Larruzea-Urkixo

ORCID-ID: https://orcid.org/0000-0002-

0559-2768

Universidad del País Vasco (UPV/EHU)

Facultad de Educación de Bilbao

Departamento de Psicología Evolutiva y de la Educación

Barrio Sarriena $\mathrm{s} / \mathrm{n}$

48940 Leioa (Spain)

E-mail: nlarucea002@ikasle.ehu.eus nerealar2@gmail.com

Olga Cardeñoso Ramírez

ORCID-ID: http://orcid.org/0000-0003-

4418-0405

Universidad del País Vasco (UPV/EHU)

Facultad de Educación de Bilbao

Departamento de Psicología Evolutiva y de

la Educación

Barrio Sarriena s/n 
48940 Leioa (Spain)

E-mail: mariaolga.cardenoso@ehu.eus

ocardenoso@gmail.com

Nahia Idoiaga Mondragon

ORCID-ID: https://orcid.org/0000-0003-

0345-8570

Universidad del País Vasco (UPV/EHU)

Facultad de Educación de Bilbao

Departamento de Psicología Evolutiva y de

la Educación

Barrio Sarriena s/n

48940 Leioa (Spain)

E-mail: nahia.idoiaga@ehu.es

nahia.idoiaga@gmail.com

Fecha Recepción del Artículo: 12. Enero. 2019

Fecha Modificación del Artículo: 18. Mayo. 2019

Fecha Aceptación del Artículo: 7. Junio. 2019

Fecha Revisión para Publicación: 23. Junio. 2019 